\title{
Statistical downscaling of monthly mean North Atlantic air-pressure to sea level anomalies in the Baltic Sea
}

\author{
By HAUKE HEYEN*, EDUARDO ZORITA ${ }^{\dagger}$ and HANS VON STORCH, Max-Planck-Institut für \\ Meteorologie, Bundesstraße 55, D-20146 Hamburg, Germany
}

(Manuscript received 23 December 1994; in final form 6 June 1995)

\begin{abstract}
The term "downscaling" describes a procedure in which information about a process with a certain characteristic scale is derived from other processes with larger scales. The present paper identifies a relationship between the main components of the North Atlantic air-pressure anomalies at sea-level (characteristic length-scale $>1000 \mathrm{~km}$ ) and the sea level anomalies at several Baltic Sea gauges $(10 \mathrm{~km}-100 \mathrm{~km})$ in winter. Monthly means from 20 observed winters are used to fit a statistical model that describes the dependence between both parameters. Further observations from this century are used to validate this model, which is able to estimate sea level anomalies from the air-pressure field to a good level of approximation. Sea level anomalies with periods from months to decades are reproduced well. As main forcing for the sea level anomalies, wind-stress with a strong zonal component is identified. For the past 89 years, we found that a slight decrease of mean sea level was induced by air-pressure. A slight increase is found when air-pressure from a GCM "greenhouse" experiment is downscaled.
\end{abstract}

\section{Introduction}

General Circulation Models (GCMs) are a powerful tool in studies of the global climate. These models operate with typical grid sizes (or equivalent wave numbers) of about $500 \mathrm{~km}$ by $500 \mathrm{~km}$ and therefore cannot resolve orography or energy fluxes on smaller scales. While GCMs are able to reproduce "large-scale" climate features to a good level of approximation ("large-scale" refers to processes with a characteristic length scale of several grid lenghts) values at single grid points are questionable and should not be interpolated to derive information about processes on smaller scales (Robinson and Finkelstein, 1991). However, applications such as climate impact research need

\footnotetext{
* Corresponding author.

+ Current affiliation: Laboratoire d'Océanographie Dynamique et de Climatologie, Université Pierre et Marie Curie, 4 Place Jussieu Tour 14, F-75252 Paris Cedex 05, France.
}

information on smaller scales. Thus some means must be found to relate large-scale information to small-scale or "local" parameters.

There are several possible approaches to do so: The "numeric" approach "nests" a finer resolved model (Limited Area Model) into the coarser one. In the "analogue" approach typical large-scalesituations and their associated local parameters are catalogued; to derive a local parameter from a new large-scale-situation, one selects a similar large-scale-situation from the catalogue and looks for its associated local parameters. In this paper we use a "statistic" approach that looks for a linear correlation between the large-scale- and the local parameter. The steps in statistical downscaling are as follows. (1) Identify a large-scale parameter $G$ which controls the local parameter $L$. If the intent is to calculate $L$ for climate experiments, $G$ should be simulated well by climate models. (2) Find a statistical relationship between $L$ and $G$. (3) Validate the relationship with independent data. (4) If the relationship is confirmed, $G$ can be derived from GCM-experiments to estimate $L$. 
The features of statistical downscaling are as follows. (a) Only small computers are needed to do the calculations. (b) There is no need for detailed knowledge about the physical processes. (c) Long and homogenous time series are needed for fitting and confirming the statistical relationship. Unfortunately such series exist for a few parameters only.

In Section 2, a description of the statistical model is given. Section 3 contains information about the used datasets. Results are given in the following sections: fit and validation of the statistical model (Section 4), a discussion about its physical implications (Section 5) and an example for downscaling GCM-data (Section 6). A brief summary and a discussion of the results complete this paper.

\section{The statistical model}

\subsection{Filtering the "main signals" from the data}

Surely we cannot expect to find a relationship between all details of the large-scale- and the local parameter, but we can try to relate the main signals of both. By means of empirical orthogonal functions (EOFs) we separate these "main signals" from noise and details at single grid points or stations. Restricting the following correlation on the main signals yields advantages: (i) Random noise which might worsen a correlation between parameters is discarded. (ii) Details that might veil the main modes of variability are discarded. Details in GCM-data are questionable anyway. Discarding details in the observational data makes possible to compare it with the GCM-data. (iii) The amount of data is reduced.

Let $L(x, t)$ be the observation of parameter $L$ at station $x$ and time $t$. Let $m$ be the number of stations and $n$ the number of timesteps. Using EOFs $\boldsymbol{L}$ is expanded into

$$
L(x, t)=\sum_{i=1}^{m}\left[\Lambda_{i}(x) * \lambda_{i}(t)\right],
$$

where $\boldsymbol{\Lambda}_{i}$ is the $i$ th "pattern" and $\lambda_{i}$ the $i$ th "time series". Patterns and time series fulfill the orthogonality conditions: If we normalize the timeseries it is $\left\langle\boldsymbol{\Lambda}_{i}, \boldsymbol{\Lambda}_{j}\right\rangle=\sigma_{i}^{2} * \delta_{i j}$ and $\left\langle\lambda_{i}, \lambda_{j}\right\rangle=\delta_{i j}$.

A measure of the "importance" of a pattern is its amount of "explained variance": Let us define the variance of a $m * n$ matrix of observations $L(x, t)$ as

$$
\operatorname{Var}(\boldsymbol{L})=\frac{1}{n-1} \sum_{i=1}^{m} \sum_{j=1}^{n}[L(i, j)-\bar{L}(i)]^{2},
$$

with

$\bar{L}(i)=\frac{1}{n} \sum_{j=1}^{n} L(i, j)$

A special property of EOFs is that Var $\left(\boldsymbol{L}-\boldsymbol{\lambda}_{i} * \boldsymbol{\Lambda}_{i}\right)$ is a minimum for $i=1$. In words, $\lambda_{1} * \Lambda_{1}$ comes closest to the observations $L$, it "explains the largest portion of variance". $\lambda_{2} * \Lambda_{2}$ comes next closest by being orthogonal to the former pair, and so on. Generally, $\boldsymbol{L}$ can be approximated well by regarding the leading $k$ patterns and timeseries only, with $k \leqslant m$. In the following, we assume that the "signals" or "main modes of variability" lie within a "signal-subspace" that is spanned by the $k$ leading patterns that explain a high $\%$ of variance.

\subsection{Correlation between local and large-scale parameter}

A canonical correlation analysis (CCA) is conducted to find a connection between the signalsubspaces $\boldsymbol{L}_{\mathrm{S}}$ and $\boldsymbol{G}_{\mathrm{S}}$ of both parameters. The CCA expands two simultaneously observed parameters $\boldsymbol{L}_{\mathrm{S}}$ and $\boldsymbol{G}_{\mathrm{S}}$ into

$$
\begin{aligned}
& L_{\mathrm{S}}(x, t)=\sum_{i=1}^{I}\left[\Lambda_{\mathrm{C} i}(x) * \lambda_{\mathrm{C} i}(t)\right], \\
& G_{\mathrm{S}}(x, t)=\sum_{i=1}^{I}\left[\Gamma_{\mathrm{C} i}(x) * \gamma_{\mathrm{C} i}(t)\right],
\end{aligned}
$$

where $\boldsymbol{\Lambda}_{\mathrm{C} i}, \boldsymbol{\Gamma}_{\mathrm{C} i}$ are the $i$ th-CCA-patterns and $\lambda_{\mathrm{C} i}, \gamma_{\mathrm{C} i}$ are the $i$ th-CCA-time series. The patterns and time series fulfill the following conditions.

- With $\rho_{i}$ being the correlation between the $i$ th pair of normalized time series, we have:

$$
\begin{aligned}
& \left\langle\gamma_{\mathrm{C} i}, \gamma_{\mathrm{C} j}\right\rangle=\left\langle\lambda_{\mathrm{C} i}, \lambda_{\mathrm{C} j}\right\rangle=\delta_{i j}, \\
& \left\langle\gamma_{\mathrm{C} i}, \lambda_{\mathrm{C}_{j}}\right\rangle=\rho_{i} * \delta_{i j} .
\end{aligned}
$$

- $\lambda_{\mathrm{Cl}}$ and $\gamma_{\mathrm{Cl}}$ have maximum possible correlation, $\lambda_{\mathrm{C} 2}$ and $\gamma_{\mathrm{C} 2}$ have next highest correlation by being orthogonal to the former pair, and so on. 
- $\lambda_{\mathrm{C} i}$ and $\gamma_{\mathrm{C} i}$ minimize

$$
\begin{aligned}
& \left\|\boldsymbol{L}_{\mathrm{S}}(t)-\sum_{i=1}^{I}\left[\boldsymbol{\Lambda}_{\mathrm{C} i}(x) * \lambda_{\mathrm{C} i}(t)\right]\right\|, \\
& \left\|\boldsymbol{G}_{\mathrm{S}}(t)-\sum_{i=1}^{I}\left[\boldsymbol{\Gamma}_{\mathrm{C} i}(x) * \gamma_{\mathrm{C} i}(t)\right]\right\| .
\end{aligned}
$$

For a more detailed description of the CCA, see, e.g., Barnett and Preisendorfer (1987).

\subsection{Deriving local data from the large-scale parameter}

We used the same strategy as von Storch et al. (1993) or Cui et al. (1995).

Let $\boldsymbol{G}_{\mathrm{S}}$ and $\boldsymbol{L}_{\mathrm{S}}$ be the observed signal-subspaces of the large-scale and the local parameter which have already been correlated to each other and let $\boldsymbol{G}^{\prime}, \boldsymbol{L}^{\prime}$ be independent data (e.g., observations from another period) with the signal-subspaces $\boldsymbol{G}_{\mathrm{S}}^{\prime}$ and $\boldsymbol{L}_{\mathrm{S}}^{\prime}$. Under certain assumptions it is now possible to estimate $\boldsymbol{L}_{\mathrm{S}}^{\prime}$ from $\boldsymbol{G}_{\mathrm{S}}^{\prime}$ :

Firstly suppose that $\boldsymbol{G}_{\mathrm{S}}$ and $\boldsymbol{G}_{\mathrm{S}}^{\prime}$ share the same $\Gamma_{C}$. According to formula (2) projecting $\boldsymbol{G}^{\prime}$ (or $\boldsymbol{G}_{\mathrm{S}}^{\prime}$ ) onto $\Gamma_{C}$ yields:

$G^{\prime}(x, t)=\sum_{i=1}^{I}\left[\Gamma_{\mathrm{C} i}(x) * \gamma_{\mathrm{C} i}^{\prime}(t)\right]+\varepsilon$,

where we minimize the error $\varepsilon$ via a least square method to calculate the new time series $\gamma_{C}^{\prime}$.

Assuming that $\gamma_{\mathrm{C}}^{\prime}$ is correlated to a time series $\lambda_{\mathrm{C}}^{\prime}$ in the same manner as $\gamma_{C}$ and $\lambda_{C}$ were, we estimate $\lambda_{\mathrm{C}}^{\prime}$ according to (3) as $\lambda_{\mathrm{C}}^{\prime}=\rho_{i} * \gamma_{\mathrm{C}}^{\prime}$.

Finally, if $\boldsymbol{L}^{\prime}$ and $\boldsymbol{L}_{\mathrm{S}}$ share the same $\boldsymbol{\Lambda}_{\mathrm{C}}$, we write, neglecting $\varepsilon$ :

$$
\begin{gathered}
L_{\mathrm{S}}^{\prime}(x, t)=\sum_{i=1}^{h \leqslant I}\left[\Lambda_{\mathrm{C} i}(x) * \lambda_{\mathrm{C} i}^{\prime}(t)\right] \\
=\sum_{i=1}^{h \leqslant I}\left[\Lambda_{\mathrm{C} i}(x) * \gamma_{\mathrm{C} i}^{\prime}(t) * \rho_{i}\right] .
\end{gathered}
$$

\section{The datasets}

In the present paper, we used winter data from the months December, January and February, because this is the season with the largest anomalies. The analysis could, of course, also be done for other seasons. Note that, though the
Baltic is usually ice-covered in winter north of $59^{\circ} \mathrm{N}$, gauge data should be generally trustworthy (see Omstedt and Nyberg, 1991).

\subsection{Baltic Sea sea level data}

We used data from the British Oceanographic Data Center (BODC) and selected 23 stations (Fig. 1) which reported between 1899 and 1987. The most observations fell within 1951 to 1970 , so we selected data from this period to establish the statistical relationship between sea level and airpressure.

The time series at Ölands Norra Udde, Gdansk and Swinoujscie possess sudden discontinuities, probably because the reference level at these stations was changed during the period of observation. We obtained "homogenous" anomaly time series for these stations by calculating the anomalies for the periods before and after the discontinuity separately.

For reasons that are stated below, the observational records contain trends. We define a trend as a variation with a period much longer than the time series. Such a variation appears as linear de- or increase in the data. To make sure that the correlations are not dominated by common linear trends, we subtract linear trends from the sea level data before correlating it with the air-pressure.

\subsection{Sea-level air-pressure data}

We used air-pressure as the large-scale parameter $G$ for three important reasons: Firstly, in addition to the effect of pressure itself, this parameter contains information about wind strength, wind direction and advection of other parameters, which all influence sea level, thus making it likely that we will find a connection between air-pressure and sea level. Secondly, we have long homogenous time series available. Lastly, large-scale airpressure fields are well simulated by GCMs.

We utilized data from the National Meteorological Center (a critical review of the NMC-dataset was given by Trenberth and Paolino, 1980). We interpolated the data on a $5^{\circ}$ by $5^{\circ}$ grid (this was done for reasons of comparability, as we also interpolated the GCM-data on such a grid) and selected a box within $70^{\circ} \mathrm{W}$ to $25^{\circ} \mathrm{E}$ and $30^{\circ} \mathrm{N}$ to $70^{\circ} \mathrm{N}$. A wide westward extension was chosen since we expected the gulf stream system and the west wind drift to influence Baltic weather and climate, an eastward extension was hindered by 
missing observations. For December 1944 we have no observations at all. We subtracted a linear trend at each gridpoint before correlating the airpressure to the sea level.

As climate experiment we choose the "early industrial" 150 -year integration carried out by Cubasch et al. (1995) with a global coupled oceanatmosphere model (ECHAM1/LSG). The first 50 years of this experiment (years " 40 " to " 90 ") were forced with observed atmospheric $\mathrm{CO}_{2}$ concentrations from 1935 to 1985 , afterwards a continous yearly $\mathrm{CO}_{2}$-increase of about $1.3 \%$ was assumed according to estimates of the Intergovernmental Panel on Climate Change. We obtained the air-pressure anomalies of the simulation by calculating monthly means for the first 50 years of the experiment and then subtracting these means from the entire 150 years of the experiment.

\subsection{Other datasets}

The air-pressure field contains indirect information about other processes, therefore it is not clear which mechanism really forces the sea level variations. In an attempt to identify the forcing we utilize several other datasets. These include the following.

- Precipitation: observational data collected by the National Center of Atmospheric Research. We selected 30 stations which reported between 1952 and 1970 , all of them within the water catchment area of the Baltic Sea.

- Sea level in the Skagerrak and German Bight: We used the BODC dataset and selected 8 stations, 6 of them in the German Bight, 2 of them in the Skagerrak. The observations fell within 1951 to 1970.

- Wind-stress: From daily air-pressure data (NMC dataset) we estimated the wind-stress with an empirical bulk formula by Duun-Christensen (1975).

\section{Fit and validation of the statistical model}

\subsection{Main modes of sea level-and air-pressure anomalies}

After calculating anomalies and subtracting linear trends, we perform an EOF-analysis for the period 1951 to 1970 and obtain insight into the

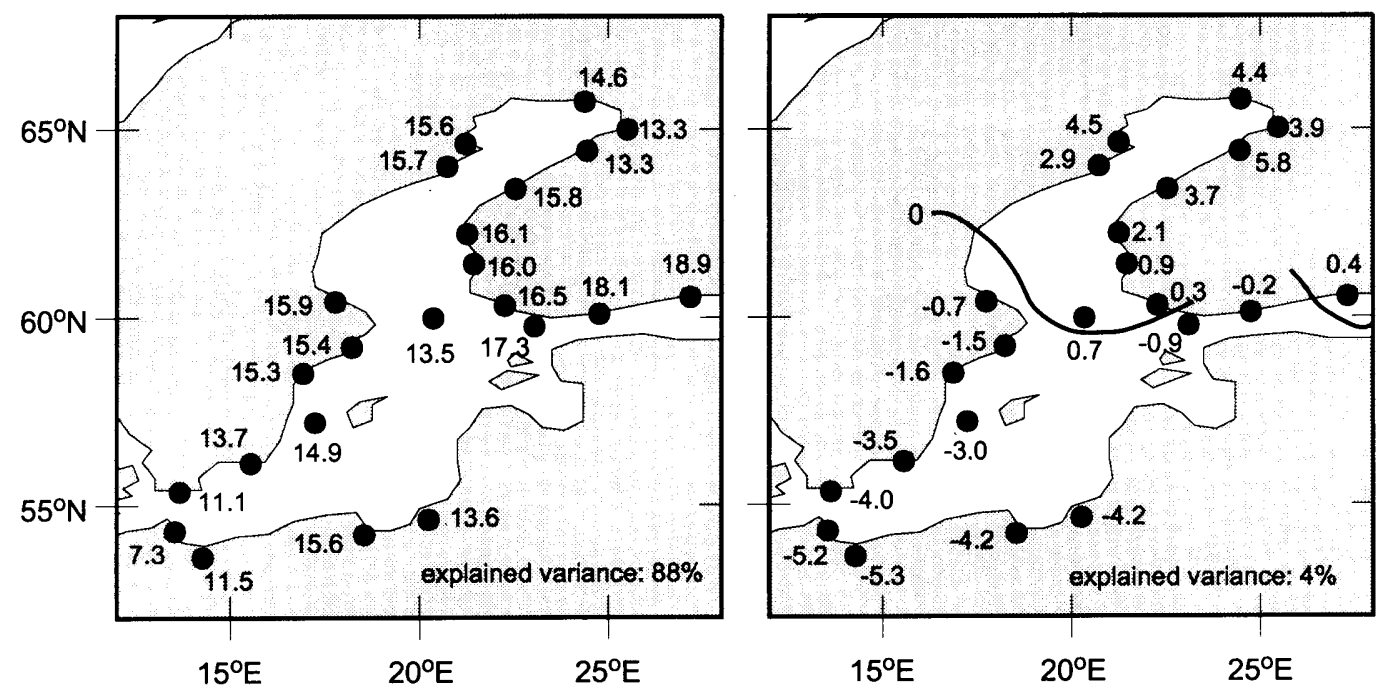

Fig. 1. The leading two sea level EOF-patterns. The patterns were derived for the winter months 1951 to 1970 and show anomalies in $\mathrm{cm}$. To improve clarity, we added an imaginary zero-line in the second pattern. Beginning in Sweden and proceeding clockwise, the 23 stations are: Sweden: Ystad, Kungholmsfort, Ölands Norra Udde, Landsort, Stockholm, Björn, Ratan, Furögrund; Finland: Kemi, Oulu, Raahe, Pietarsaari, Kaskinen, Mantyluoto, Turku, (two stations situated offshore:) Degerby and Hanko, Helsinki, Hamina; Russia: Kaliningrad; Poland: Gdansk, Swinoujscie; Germany: Sassnitz. 
main modes of variability of each parameter. Since we operate with normalized EOF-time series, the obtained patterns show typical anomalies in $\mathrm{cm}$ or hPa.

Two main modes of winter Baltic Sea sea level variations are shown in Fig. 1. The leading EOFpattern describes a simultaneous rise (fall) of sea level with an amplitude of about $10 \mathrm{~cm}$ in the southern, $15 \mathrm{~cm}$ in the Northern and $18 \mathrm{~cm}$ in the eastern Baltic Sea. This mode is dominant, since it explains already $88 \%$ of variance. The second pattern describes an opposite movement of sea level at southern and northern gauges (a "seesaw") and explains $4 \%$ of variance. Higher indexed patterns are more complex and explain a considerable amount of variance only for single stations.

The leading air-pressure EOF-pattern (not shown) describes a positive (negative) anomaly above Greenland and a negative (positive) anomaly above the Azores. This mode, which explains $35 \%$ of variance, is often referred to as the "North Atlantic Oscillation" (NAO) (Van Loon and Rogers, 1978). The second mode describes simultaneously rising (falling) air-pressure above the entire North Atlantic with its center west of Ireland. It accounts for $25 \%$ of variance. The higher indexed modes are more complex, but are still large-scale and explain a considerable amount of variance, as well.

\subsection{Correlation between sea level and air-pressure}

We selected the leading two sea level and the leading four air-pressure EOF-patterns to span the signal-subspaces. These explain $92 \%$ of sea level and $85 \%$ of air-pressure variance within the fitting period. Higher indexed patterns were discarded; in case of sea level, they describe only details at single stations. In case of air-pressure they explain less than $5 \%$ of variance each and thus do not belong to the "main modes" that are modeled by GCMs (in addition it can be shown that higher indexed patterns possess no additional information that improve the correlation any more).

A CCA between both parameters signal-subspaces reveals two pairs of patterns (Fig. 2). The first pair shows an air-pressure anomaly of about $4 \mathrm{hPa}$ above the Irish Sea and a southwest-northeastern gradient from $-1 \mathrm{hPa}$ to $-4 \mathrm{hPa}$ above the Baltic Sea. Its time series is correlated with 0.8 to the time series of a sea level pattern that describes a simultaneously rise of all Baltic gauges.
The second pair shows a NAO in the air-pressure pattern and an opposite movement of southern and northern gauges for sea level. The associated time series are correlated with 0.5 .

Note that the sea level CCA-patterns closely resemble the EOF-patterns. It is due to this coincidence that the sea level CCA-patterns explain nearly maximal variance. Together with the good correlation for the first pair this should enable us to estimate sea level variations from the airpressure field to a good level of approximation.

\subsection{Validating the relationship with observational data}

To validate the obtained relationship we downscale air-pressure anomalies from the periode 1899 to 1987 using formula (4) and compare the estimated sea level anomalies with the observed ones. We find that the degree of correlation varies from station to station and also from year to year (Table 1). The latter is certainly due to the fact that the explained variance of the utilized CCA-patterns varies considerably with time, too (Table 1). In addition, it can be shown that the quality of the relationship also varies, i.e., that we obtain other CCA-patterns if we select another fitting period (such patterns are similar, but not identical to that from Fig. 2).

Table 1 depicts two more important points: Firstly, that the leading sea level CCA-pattern explains about $90 \%$ of variance for all periods. This means that estimates made with this leading pattern will always include the biggest portion of sea level variations. Secondly, the second CCApair improves the overall correlation only for the fitting period. An explanation for this was suggested by J. Dippner (personal communication): from around 1960 to 1970 , the NAO-index was outstanding low, leading to weak zonal flows (Rogers, 1984). As we will see later, zonal windstress associated with the first air-pressure CCApattern forces the first sea level CCA-pattern, while meridional wind-stress associated with the second CCA-air-pressure pattern forces the seesaw-mode. A low NAO-index and a weak zonal circulation thus probably increase the relative importance of the second pair of CCA-patterns during the fitting period.

For the two above mentioned reasons we will use only the first CCA-pair in all following estimations. 

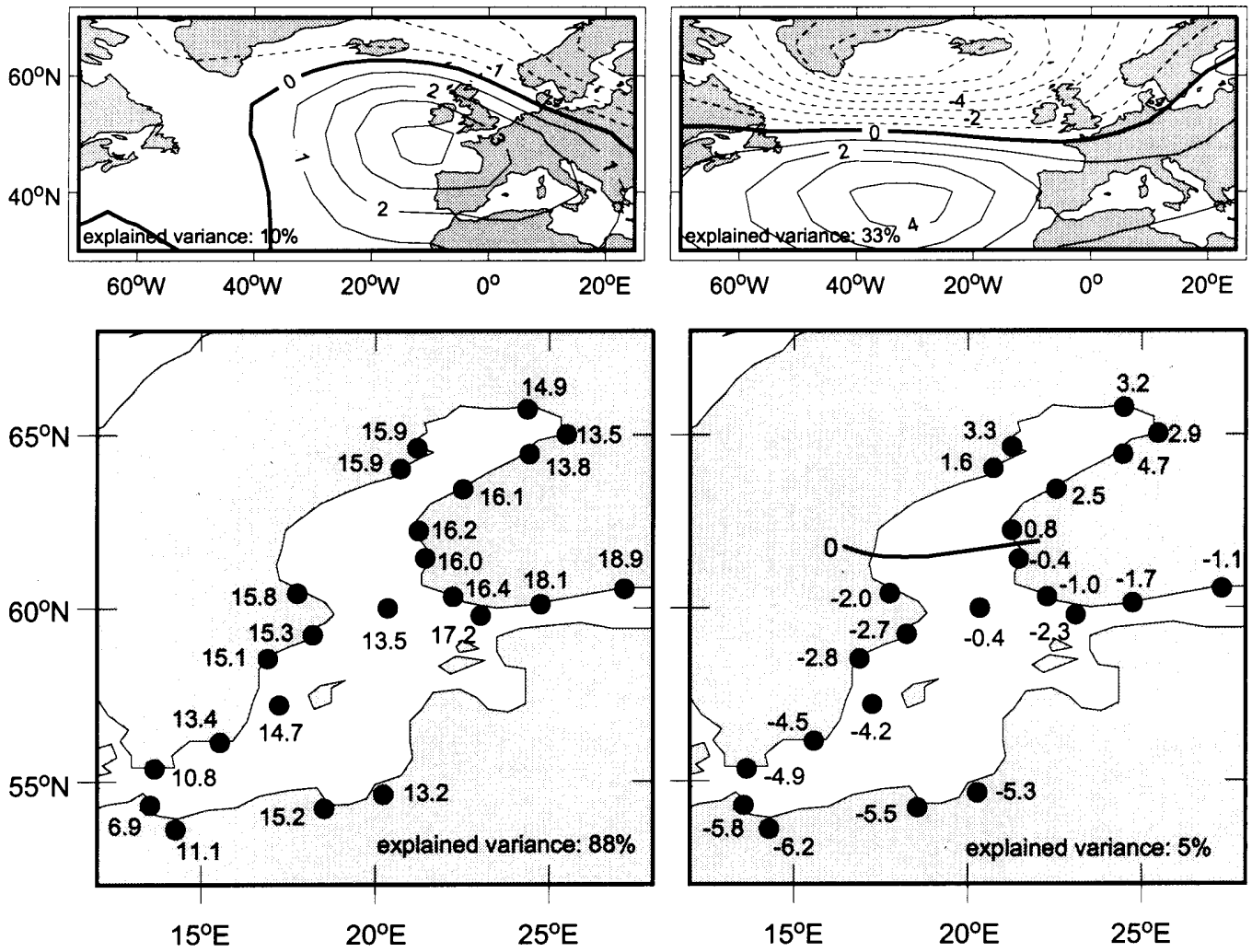

Fig. 2. The first (left) and second (right) pair of CCA-patterns. Each pair depicts anomalies in hPa and $\mathrm{cm}$ that belong together, the associated time series are correlated with 0.8 (left) and 0.5 (right).

Table 1. Left column. Different 15 -year periods; years used for fitting (top), other observational years (mid), GCM-years (bottom). Mid columns. Explained variance of the 1st/2nd CCA-pair in that period. Right columns. Correlations between observations and estimates made with one/both leading CCA-patterns

\begin{tabular}{crcccccc}
\hline Period & Pressure & Sea level & Ystad & Swin. & Hels. & Raahe & All St. \\
\hline $1951-1965$ & $9 \% / 37 \%$ & $90 \% / 4 \%$ & $0.39 / 0.57$ & $0.36 / 0.64$ & $0.61 / 0.71$ & $0.66 / 0.68$ & $0.60 / 0.69$ \\
$1901-1915 *$ & $18 \% / 24 \%$ & $88 \% / 7 \%$ & $0.42 / 0.41$ & $0.50 / 0.52$ & $0.74 / 0.71$ & $-/-$ & $0.67 / 0.63$ \\
$1926-1940$ & $17 \% / 32 \%$ & $86 \% / 2 \%$ & $0.19 / 0.24$ & $0.30 / 0.47$ & $0.70 / 0.65$ & $0.68 / 0.60$ & $0.63 / 0.56$ \\
$1971-1985$ & $22 \% / 28 \%$ & $90 \% / 3 \%$ & $0.52 / 0.52$ & $0.50 / 0.57$ & $0.82 / 0.80$ & $0.83 / 0.78$ & $0.81 / 0.77$ \\
$61-75$ & $8 \% / 19 \%$ & $-/-$ & $-/-$ & $-/-$ & $-/-$ & $-/-$ & $-/-$ \\
$161-175$ & $7 \% / 19 \%$ & $-/-$ & $-/-$ & $-/-$ & $-/-$ & $-/-$ \\
\hline
\end{tabular}

* Estimation for 13 stations. 
Fig. 3 depicts observed and estimated (with one CCA-pair) sea level anomalies. The estimates generally have the correct sign. In addition, they contain variations on the decadal time scale that are in good correspondance with the observations. Indeed a comparision between the power spectrum of observed and estimated sea level reveals no deficiencies of the estimated variability for periods
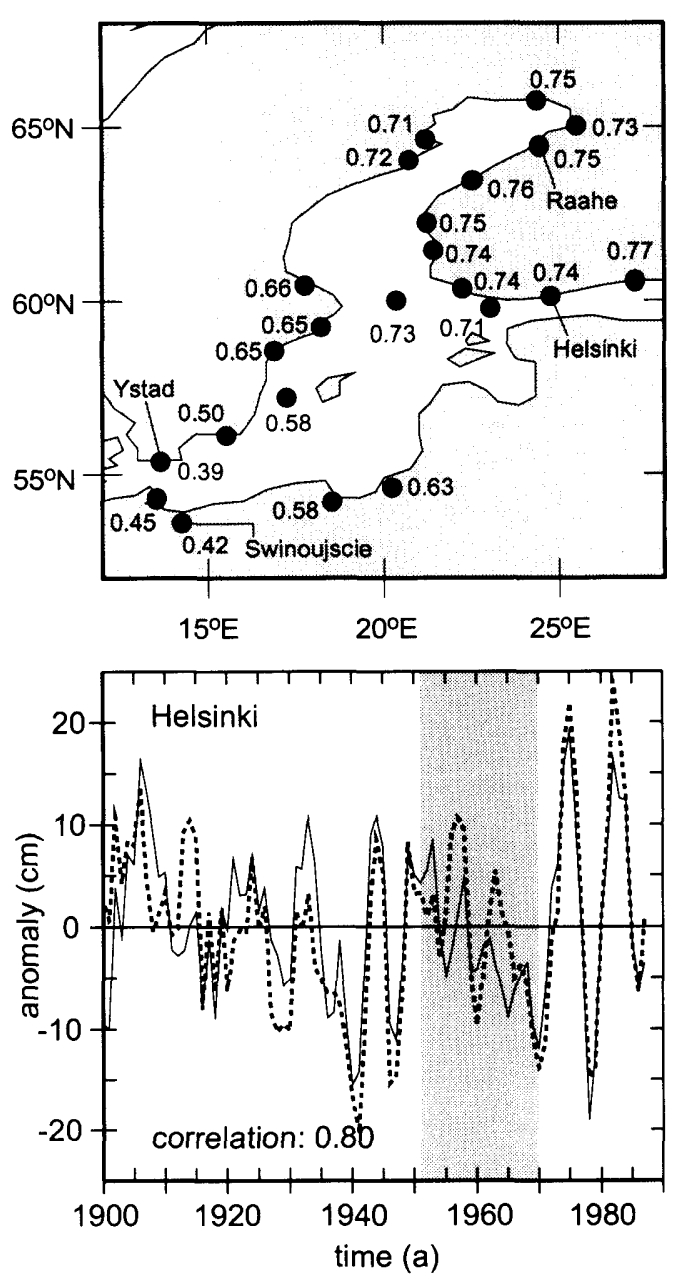

Fig. 3. Detrended winter air-pressure observations within 1899 and 1987 are downscaled to derive sea level anomalies. These are compared with detrended sea level observations: The map (top) depicts the local correlation between estimated and observed monthly means. The graph (below) shows estimated (solid line) and observed (dashed line) anomalies for one selected station, smoothed by a 3 year-running mean. up to 44 years ( 44 years are the "safe" upper end of the spectrum since the time series is only twice as long). This is not self-evident, since the model was fitted with 20 years of detrended data and thus is familiar with monthly and yearly variations only. That it produces decadal variations though, makes us believe that it may guess trends in a realistic manner, too.

The discrepancy between downscaled and observed values before 1920 (Fig. 3) is real, since related problems had been noted before (personal communication with J.-E. Lundqvist, Swedish Meteorological and Hydrological Institute), but the reason is unknown. The decreasing local correlations between estimated and observed values for stations situated in the southwest (Fig. 3, top left) may be due to a more complex behaviour of sea level there: In this region in- and outflow situations lead to high velocities and strong haline gradients. This results in a more complex behaviour of the gauges situated there, that is not represented within the selected signalsubspace.

\section{Physical implications of the statistical model}

\subsection{Physical links between air-pressure and sea level}

We revealed a statistical relationship between large-scale air-pressure and local sea level. Now we ask for the physical meaning of this relationship. The leading pair of CCA-patterns (Fig. 2, top) suggests the following interpretation (for clarity we suppress the term "anomalies"): Northwestern winds bring marine (moist) air to Scandinavia, inducing precipitation and enhanced river-runoff. The wind-stress piles up water in the German Bight, creating a sea level gradient directed downward into the Baltic Sea. The low pressure above the Baltic Sea leads to an inflow of water through the Skagerrak or hinders an outflow. All these phenomenon tend to increase the Baltic Sea sea level.

It remains unclear whether all of the above stated phenomena are of similar importance for sea level, or whether some are negligible. To separate the more important mechanisms from those that are less important we make the following hypothesis: 
- If a parameter forces sea level variations we assume that a high percentage of it will be highly correlated to sea level in a spatial sensful context. By "spatial sensful context" we mean that it is not necessary that all, e.g., rain stations, explain much variance and are highly correlated with sea level; instead, this is necessary only for those stations which are located within the water catchment area of the Baltic Sea.

We proceed as following: Let $\lambda_{\mathrm{C} i}$ be the $i$ thCCA-time series of the sea level anomalies $L$, and $D$ be the anomalies of the forcing parameter of interest. Assuming high correlation between $\boldsymbol{L}$ and $\boldsymbol{D}$, we define "associated patterns" $\boldsymbol{\Delta}_{\mathrm{A} i}$ such that $\Delta_{\mathrm{A} i}$ minimize $\varepsilon^{2}$ in

$D(x, t)=\sum_{i=1}^{I}\left[\Delta_{\mathrm{A} i}(x) * \lambda_{\mathrm{C} i}(t)\right]+\varepsilon$

In our case $I=1$, since we have one sea level pattern. Thus we find one pattern $\Delta_{\mathrm{A} i}$ that possesses the same time series as $\boldsymbol{\Lambda}_{\mathrm{C} i}$. If $\boldsymbol{\Delta}_{\mathrm{A} i}$ explains a considerable portion of the variance of $\boldsymbol{D}$ it means that "a considerable portion of $\boldsymbol{D}$ is correlated with 1.0 to $88 \%$ of sea level variance". Depending on the portion and its spatial distribution we will consider $\boldsymbol{D}$ as an important forcing for $\boldsymbol{L}$.

Note that it is not possible to strictly separate the individual forcing mechanisms from each other: As the air-pressure field did contain indirect information about precipitation, for example, the precipitation field yields information about the air-pressure. Thus the following results should be understood as estimates, though the final result, that wind-stress is the most importing forcing for Baltic Sea sea level variations, corresponds with the observations (Dietrich, 1951).

5.1.1. Precipitation and river-runoff. The precipitation pattern that is associated with the leading sea-level CCA-pattern depicts three stations in Finland that explain about $15 \%$ of precipitation variance each (not shown). At other stations around the Baltic Sea (and within the water catchment area) often $0 \%$ of precipitation is correlated with the sea level variations. Thus we believe that precipitation is not important for sea level variations.

Concerning river-runoff, we have no data available to estimate its relative importance. We believe, though, that its importance will be small since the runoff is generally small in winter (this might also be the explanation for the nonimportance of precipitation in this season). In other seasons, particular in spring (begin of melting), river-runoff might become significant.

5.1.2. Inverse barometrical effect. We expect the sea level to rise below low air-pressure and vice versa. For an infinite ocean, this relationship is well known and linear, but since the Baltic Sea is a narrow basin with a narrow opening to the North Sea (which is itself not "infinite") the situation is more complex. Thus we can only speculate about the impact of the inverse barometrical effect: referring to the first sea level CCA-pattern we see that typical air-pressure anomalies of about $\pm 4 \mathrm{hPa}$ occur above the Baltic Sea. If we assume equilibrium and infinity as a first rough approximation, this would induce sea level anomalies of about $\mp 4 \mathrm{~cm}$ (since water transport through Kattegat and Skagerrak can be very effective (Wyrtki, 1955), the orography sets no obstacle for an equilibrium between pressure and sea level in the monthly mean. It is the complex interference of other factors as wind that calls an equilibrium state into question). Thus the inverse barometric effect could account for a few $\mathrm{cm}$ anomaly in the first sea level CCA-pattern.

5.1.3. Wind-stress. In the transition area between North Sea and Baltic Sea, up to $40 \%$ of wind-stress variance is associated with the leading sea level CCA-pattern (Fig. 4). Obviously westsouthwesterly winds push water into the Skagerrak, induce an inflow into the Baltic Sea or suppress an

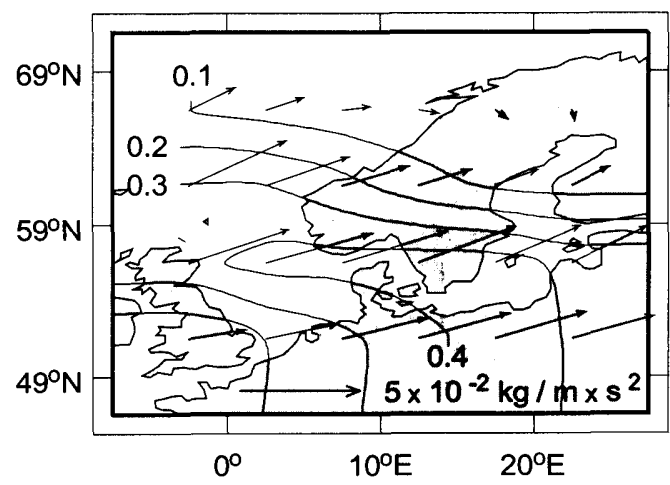

Fig. 4. Vectors indicate the wind-stress that is associated with the first sea level CCA-pattern. Isolines depict the amount of variance that is explained by the associated wind-stress. 
outflow, and thus lead to a simultaneous rise at all Baltic Sea gauges.

5.1.4. Sea level in the German bight. German Bight sea level anomalies of about $+10 \mathrm{~cm}$ go along with Baltic Sea sea level anomalies between $+10 \mathrm{~cm}$ and $+18 \mathrm{~cm}$ (Fig. 5). The associated anomalies in the German Bight explain up to $50 \%$ of variance, thus there is a close relationship between sea level in both regions.

5.1.5. Summary. We found a big portion of west-southwesterly wind-stress and of German Bight sea level associated with the leading sea level CCA-pattern. Indeed it is probable that German Bight sea level itself is a result of wind-stress. To determine if this is the case, we performed a CCA of the combined Baltic Sea and German Bight sea level and the air-pressure. We obtained a leading pair of CCA-patterns (not shown) that correlates an entirely risen sea level at both sites with a airpressure pattern which implies westerly winds over the German Bight and Baltic Sea. Thus we believe that at least a good portion of the German Bight sea level is itself produced by zonal wind-stress.

We conclude that for winter monthly means:

- precipitation and, very likely river-runoff, have little impact on Baltic Sea sea level variations;

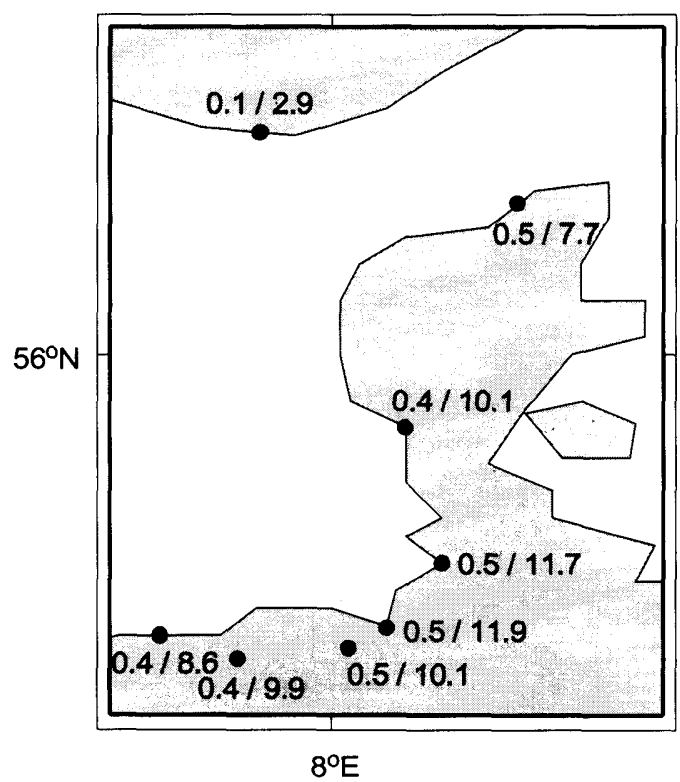

Fig. 5. Explained variances and heights $(\mathrm{cm})$ of German Bight sea level anomalies that are associated with the first Baltic Sea CCA-pattern.
- the impact of the inverse barometric effect is not known; we speculate that it might induce anomalies within the range of a few $\mathrm{cm}$;

- zonal wind-stress forces the first Baltic Sea CCA-pattern, at least to a high degree $(40 \%$ of wind-stress variance in the transition area is associated with $90 \%$ of sea level variance);

- we like to add that the same method identifies meridional wind-stress (explained variance: $30 \%$ to $40 \%$ of total wind-stress over the Baltic Sea) as dominant forcing for the second sea level CCApattern, as one would expect.

\subsection{About the origin of trends in the sea level observations}

Observations at Baltic gauges reveal considerable changes of sea level over the past 89 years. The amount of change is different from station to station, it ranges from $-0.76 \mathrm{~cm} /$ year at Pietarsaari (northern Baltic) to $+0.14 \mathrm{~cm} /$ year at Swinoujscie (southern Baltic). In this section we try to determine the origins of these changes.

The Fennoscandian Shield has been rising since the end of the last Ice Age. The center of this rising motion is situated around the northern shore of the Baltic Sea, where magnitudes up to $1 \mathrm{~cm} /$ year are observed. The line of zero rise runs through Northern Poland, Northern Germany and Denmark. We expect negative "isostatic" sea level trends for the stations that lie within the area of rise. In addition, the mean global sea level has been rising by estimated $0.2 \mathrm{~cm} /$ year (e.g., Trupin and Wahr, 1990, or Peltier and Tushingham, 1991). Thus a positive "eustatic" trend should be found at all gauges. Finally, there might be a trend due to a more recent origin, e.g., an anthropogenic climate change: it is this trend we are especially interested in.

By downscaling the observed, not detrended airpressure we obtain the sea level containing this unknown trend, which we will call the "pressure induced" trend since we derived it from pressure data. Indeed, we find a slight decrease of pressureinduced sea level over the period 1899 to 1987 (Fig. 6), but the shortness of our observational record and the decadal variability forbid us to speak of a "trend". The pressure-induced decrease of sea level ranges from about $-0.05 \mathrm{~cm} /$ year in the southern to about $-0.07 \mathrm{~cm} /$ year in the northern part of the Baltic Sea. These values are small compared to the magnitudes of the iso- and 


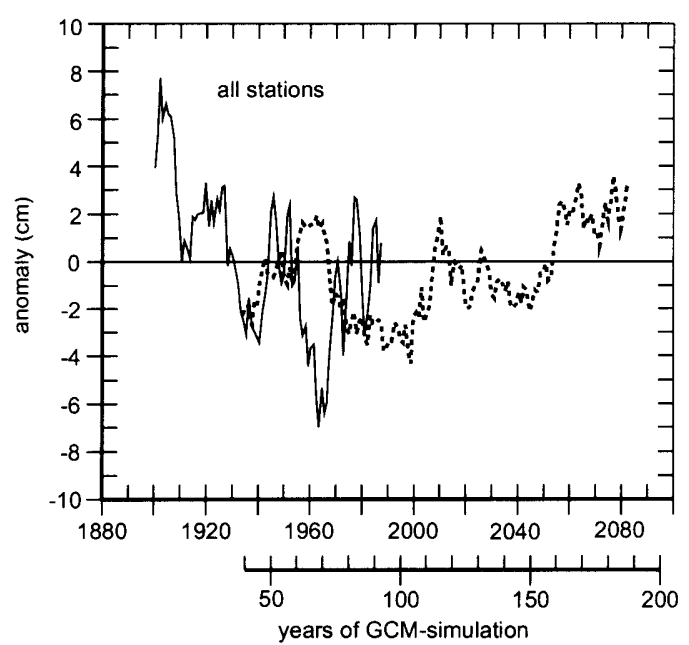

Fig. 6. Baltic Sea sea level anomalies in winter, estimated from not detrended air-pressure observations (solid line) and GCM-data (dashed line). The curves represent an average over all stations and are smoothed by a 11 yearrunning mean.

eustatic movements, even if we take into account that iso- and eustacy act opposite and will cancel each other somewhere in the southern part of the Baltic Sea. Thus we state that the sea level "trend" observed at Baltic Sea gauge stations in the past 90 years is primarily due to iso- and eustatic movements.

\section{Estimating sea level anomalies from GCM-data}

Fig. 6 depicts sea level anomalies that were downscaled from air-pressure data of a GCMclimate change experiment. We note the following.

(1) The air-pressure induced sea level anomalies show no further decrease, but seem to increase for the later years of the GCM-experiment. Over the period " 40 " to " 189 " we have a total increase of about $0.01 \mathrm{~cm} /$ year.

(2) The estimated anomalies vary on a decadal time scale, as the observations did.

(3) The variance of the guessed anomalies is smaller than that of the observed anomalies or that of the anomalies downscaled from air-pressure observations. A power spectrum reveals that the mean loss of variance is about $75 \%$, in the "best" frequency-band ( 3 to 10 years) it is about $50 \%$.
(4) For the period in which the GCM was forced with observed $\mathrm{CO}_{2}$-concentrations, the estimated anomalies behave opposite than the observed.

Points (3) and (4) need to be discussed. Let us start with the last point. Though the GCM was forced with observed atmospheric $\mathrm{CO}_{2}$-concentrations from 1935 to 1985 , the year " 40 " for example is no simulation of 1935. As climate models in general, the choosen GCM-experiment is designed only to estimate a mean development over a couple of years, not to "predict" single events. Thus a comparision between GCM and observation should be done carefully, especially with respect to the occurrence of events in fixed years. A more appropriate comparision could be: neither the observations from 1935 to 1985 , nor the "parallel" GCM-years 40 to 90 reveal a clear trend. For these periods, both curves show strong variations on a decadal and weaker variations on a yearly scale. The amplitudes of the anomalies downscaled from observations are generally stronger than the estimates from GCM-data.

Referring to the point (3), we find that the standard deviation of the monthly GCM-airpressure itself is smaller than that of the observed air-pressure. Loss of variance is a typical shortcoming of models with low resolution (the resolution of ECHAM1 is $5.6^{\circ}$ on a Gaussian grid). A comparision of the power spectrum of the observed and the GCM-air-pressure reveals that the GCM reproduces realistic variances for frequencies within 1 to 10 years, while for frequencies less than 1 year and between 10 to 30 years it underestimates the variance by about $50 \%$ and $75 \%$, respectively. This shortcoming of the GCM is surely one reason that leads to a loss of variance for the estimated sea level.

Another reason are systematic errors in the shape and location of major modes of variability that are made by ECHAM1 (Cubasch et al., 1992). Different location or different shape will result in a wrong estimation of intensity for the downscaled parameter, since a GCM-mode that is projected on a CCA-pattern with not exactly the same location or shape will lose explained variance in that pattern. Of course the chosen GCM reproduces main modes similar to the observed ones, but not identical (for example, the leading four EOFpatterns derived from the observations explain 

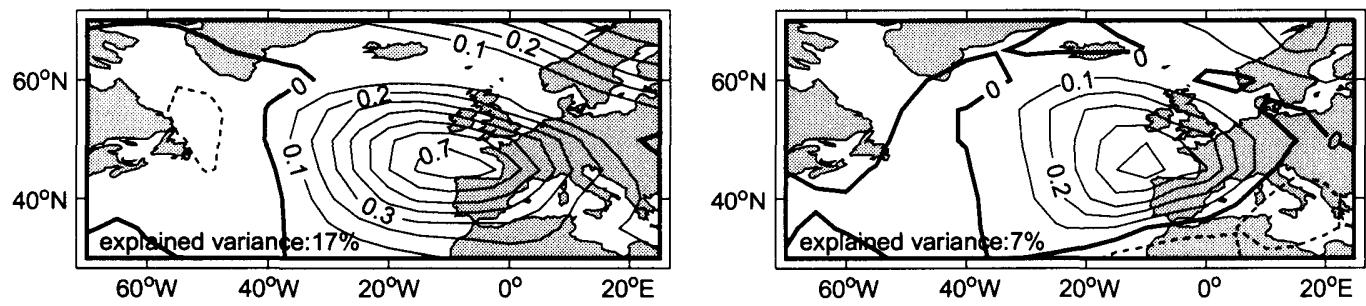

Fig. 7. Explained variance of air-pressure observations (left) and GCM-air-pressure (right), when projected onto the leading CCA-pattern.

$85 \%$ or $83 \%$ of variance for the fitting period and the entire period respectively, but only $68 \%$ of the GCM-air pressure). Since our air-pressure CCApattern represents a "minor" main mode (only $10 \%$ of explained variance), differences between the GCM-run and observation will probably even be stronger than for "main" main modes as the NAO. In Fig. 7 observations and GCM-data were projected onto the first air-pressure CCA-pattern (see also Table 1). It can be depicted that the projected GCM-data not only possesses lower intensity, but that it is distorted somehow, hinting to another location or another shape of that mode in the GCM.

If we assume that the sea level anomalies were estimated correctly, though all discussed shortcomings, the most important result is the slight increase of sea level in the long-term mean. Though this increase has a small magnitude, it has an opposite sign then in the observations from this century. For most of the Baltic this slight change will have no consequences, as iso- and eustacy will still dominate the fall or rise of coastal sea level. In the region where iso- and eustacy cancel each other, it may become important. Note that in the case of climate change this region might shift, since the magnitude of the eustatic effect might change and other effects, as thermal expansion of sea water will interfere, too.

\section{Summary and discussion}

The statistical model found a strong connection between the anomalies of the large-scale sea-level air-pressure and the locally influenced sea level in winter. Sea level anomalies may be derived from air-pressure data with satisfying results. The model is able to reproduce sea level variations on a decadal scale with good correspondence to the observed ones, though it was fitted with variations on mothly and yearly scale only. Thus reasonable results can be expected for estimates from GCMdata, too.

Due to variability on the decadal time scale and the shortness of the time series it is not serious to speak of "trends", but a slight decrease of "pressure-induced" sea level was found for the past 89 years and an increase is estimated for the climate change experiment. The magnitudes of these changes are small compared with iso- and eutatic effects, but somewhere in the southern Baltic Sea, where iso- and eustacy cancel each other, this change of sign might become important.

As our model is purely statistical, we have to check its results for physical plausibility, which implies that we must guess possible physical links between air-pressure and sea level. Regression patterns enable us to identify the wind-stress as the most important forcing for the sea level.

Let us discuss two possible weak points of statistical downscaling. Firstly, it may be argued that our model contains a lot of assumptions. Indeed, all assumptions made can be formulated as one: We assumed that currently existing structures in the air-pressure and sea level field as well as the correlations between both do not change when we go backwards or forwards in time. Indeed, this "uniformitarism" (Briffa, 1995) is wide-spread in climate research. For instance, most parameterizations employed by GCMs tacitly use empirical evidences which are thought to prevail under changed climatic conditions as well. Another example is proxy data such as temperatures derived by means of dendrochronology.

Secondly, we conclude on decadal variations using models that are derived from "high-frequency" variations only. It is not self-evident that this works, since if the mean state of a system is 
changed slowly, e.g., due to decadal variations, other equilibriums and other relationships might become valid.

In both cases we tested our assumptions by running our model with data from another period. The results show that obviously some uniformitarianism exists, even though the mean state varies considerably for different decades. That this is indeed no isolated case can be seen by similar works from Cui et al. (1995) or von Storch et al. (1993).

\section{Acknowledgements}

The idea for the present paper originates from a fruitful communication between Hans von Storch and Jan Backhaus (Institut für Meereskunde, Universität Hamburg). Financial support was provided by the European Community through project EV5V-CT91-0051. For the informative figures we like to thank Marion Grunert, for helpful comments Joachim Dippner (both MaxPlanck-Institut für Meteorologie).

\section{REFERENCES}

Barnett, T. and Preisendorfer, R. 1987. Origins and levels of monthly and seasonal forecast skills for United States surface air temperatures determined by canonical correlation analysis. Mon. Wea. Rev. 15, $1825-1850$.

Briffa, K. R. 1995. Interpreting high-resolution proxy data: the example of dendroclimatology. In: Analysis of climate variability-applications of statistical techniques (ed. H. von Storch and A. Navarra). Springer Verlag, pp. $77-94$, in press.

Cubasch, U., Hasselmann, K., Höck, H., Maier-Reimer, E., Mikolajewicz, U., Santer, B. D. and Sausen, R. 1992. Time-dependent greenhouse warming computations with a coupled ocean-atmosphere model. Clim. Dyn. 8, 55-69.

Cubasch, U., Hegerl, G., Hellbach, A., Höck, H., Mikolajewicz, U., Santer, B. D. and Voss, R. 1995. A climate change simulation starting at an early time of industrialization. Clim. Dyn., in press.

Cui, M., von Storch, H. and Zorita, E. 1995. Coastal sea level and the large-scale climate state: a downscaling exercise for the Japanese Islands. Tellus 47A, 132-144.

Dietrich, G. 1951. Oberflächenströmungen im Kattegat, im Sund und in der Beltsee. Dt. Hydrogr. Zt. 4, 129-150.

Duun-Christensen, J. T. 1975. The representation of the surface air-pressure field in a two dimensional hydrodynamic model for the North Sea, the Skagerrak and the Kattegatt. Dt. Hydrogr. Zt. 28, 97-116.
Omstedt, A. and Nyberg, L. 1991. Sea level variations during ice-covered periods in the Baltic Sea. Geophysica 27, 41-61.

Peltier, W. R. and Tushingham, A. M. 1991. Influence of glacial isostatic adjustment on tide gauge measurements of secular sea level change. J. Geophys. Res. 96, 6779-6804.

Robinson, P. J. and Finkelstein, P. L. 1991. The development of impact-oriented scenarios. Bull. Amer. Met. Soc. 4, 481-490.

Rogers, C. J. 1984. The association between the North Atlantic Oscillation and the Southern Oscillation in the northern hemisphere. Mon. Wea. Rev. 112, 1999-2015.

Trenberth, K. E. and Paolino (jr.), D. A. 1980. The northern hemisphere SLP-dataset: trends, errors and discontinuities. Mon. Wea. Rev. 108, 855-872.

Trupin, A. and Wahr, J. 1990. Spectroscopic analysis of global tide gauge sea level data. Geophys. J. Int. 100, 441-453.

Van Loon, H. and Rogers, J. C. 1978. The seesaw in winter temperatures between Greenland and Northern Europe (I). Mon. Wea. Rev. 106, 296-310.

Von Storch, H., Zorita, E. and Cubasch, U. 1993. Downscaling of global climate change estimates to regional scales: an application to Iberian rainfall in Wintertime. J. Climate 6, 1161-1171.

Wyrtki, K. 1955. Die Dynamik der Wasserbewegung im Fehmarnbelt. Kieler Meeresforschungen IX2., 155-170. 